\title{
CURE KINETICS OF EPOXY RESIN-NATURAL ZEOLITE COMPOSITES
}

\author{
B. C. Erdoğan ${ }^{*}$, A. T. Seyhan ${ }^{2}$, Y. Ocak ${ }^{1}$, M. Tanoğlu ${ }^{2}$, D. Balköse ${ }^{1}$ and S. Ülkü \\ ${ }^{1}$ Department of Chemical Engineering, Izmir Institute of Technology, Gulbahce Koyu, Urla, Izmir, Turkey \\ ${ }^{2}$ Department of Mechanical Engineering, Izmir Institute of Technology, Gulbahce Koyu, Urla, Izmir, Turkey
}

\begin{abstract}
The cure kinetics of epoxy resin and epoxy resin containing 10 mass $\%$ of natural zeolite were investigated using differential scanning calorimetry (DSC). The conformity of the cure kinetic data of epoxy and epoxy-zeolite system was checked with the autocatalytic cure rate model. The results indicated that the hydroxyl group on the zeolite surface played a significant role in the autocatalytic reaction mechanism. This group was able to form a new transition state between anhydride hardener and epoxide group. The natural zeolite particles acted as catalyst for the epoxy system by promoting its curing rate.
\end{abstract}

Keywords: autocatalytic cure rate, clinoptilolite, epoxy

\section{Introduction}

Epoxy resins are the most important thermosetting polymers widely used as matrices in reinforced composites, adhesives in the aerospace industry, surface coatings, etc. These resins have good thermal, electrical and mechanical properties, but they are brittle and have poor resistance to crack propagation [1-4]. The chemical formulation of epoxy resin is given in Fig. 1. Epoxy resins are prepared in the presence of amine and anhydride reagents. Anhydride-type epoxies reportedly give a lower exotherm during cure and generally provide better thermal stability and electrical insulation. The anhydride ring must first be opened by active hydrogen present as water or as hydroxyl compounds. The reactions of the anhydride resin with the hydroxyl group and epoxy group are given in Figs 2 and 3. The final cross-linked, phthalic anhydride-cured epoxy is shown in simplified form in Fig. 4. Epoxy resins are mixed with modifying agents, such as low molecular mass inorganic fillers, in particular zeolites and plasticizers to increase the resistance of the crack propagation. Zeolites are based on crystalline aluminosilicate minerals and are applied in many fields as catalysts and ion exchange materials $[5,6]$.

To investigate the cure kinetics of epoxy resins, many researchers proposed several techniques such as differential scanning calorimetry (DSC), Fourier transform IR (FTIR), magnetic resonance spectroscopy (NMR) and high pressure liquid chromatography (HPLC) [1]. DSC is one of the best-known methods and is mainly divided into two categories, namely isothermal and dynamic scanning $[2,4,7]$. In dynamic scanning, the sample is heated at a constant rate and the rate of energy release monitored as a function of time. The total heat of reaction $H_{\mathrm{U}}$ is calculated from Eq. (1). In isothermal scanning, the sample is heated to a constant temperature and the rate of energy release monitored until the reaction becomes completed. The isothermal heat of reaction $H_{\mathrm{T}}$ is determined from Eq. (2). In both equations, $\mathrm{d} Q / \mathrm{d} t$ represents the instantaneous heat generation rate and $t_{\mathrm{f}}$ the amount of time required to complete the reaction [8]. The rate of cure during an isothermal cure is determined by using Eq. (3). The degree of cure $(\alpha)$ is the ratio of the amount of heat<smiles>CC(C)(OCC(O)COc1ccc(C(C)(C)c2ccc(C(C)(C)c3ccc(OCC4CO4)cc3)cc2)cc1)c1ccc(OCC2CO2)cc1</smiles>

Fig. 1 The chemical formula of epoxy resin<smiles>[R]OC(=O)c1ccc(C=[PH+][c-]2oc(=O)c3ccccc3c2=O)cc1C(=O)O</smiles>

Fig. 2 The reaction of anhydride resin with hydroxyl group

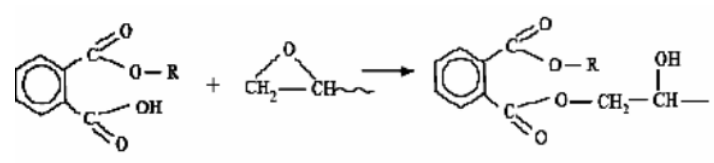

Fig. 3 The reaction of anhydride resin with epoxy group 


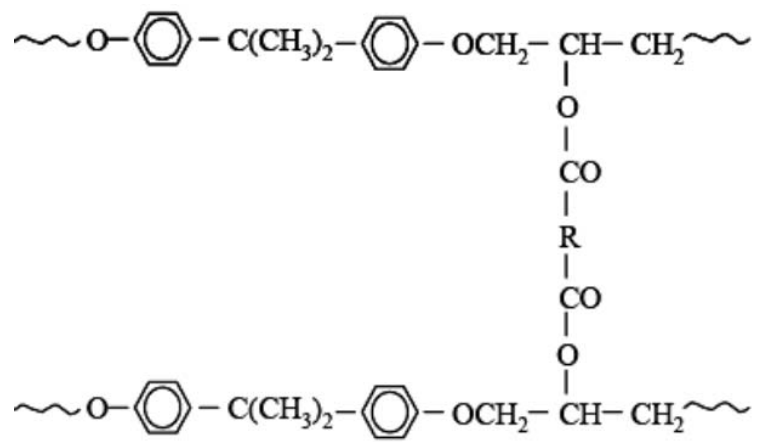

Fig. 4 The scheme of the final cross-linked, phthalic anhydride-cured epoxy

released from the beginning of the reaction until time $t$ and the total heat of the reaction. The degree of cure can be calculated by using Eq. (4). The cure kinetics of autocatalytic thermosetting resin systems are defined by Dutta-Rayan [9]. The model equation is given in Eq. (5). In this equation, $\alpha$ represents cure rate, $\alpha$ represents degree of cure, $k_{1}$ and $k_{2}$ represents reaction constants, $m$ and $n$ represents reaction orders. By assuming the zero-initial cure rate, $k_{1}$ can be eliminated from Eq. (5) and it is rewritten as Eq. (6). In order to determine the kinetic parameters $(m, n$ and $k$ ), Eqs (7)-(9) are used. In these equations, $\alpha_{\mathrm{p}}$ represents degree of cure at the exothermic peak and $\dot{\alpha}_{\mathrm{p}}$ represents cure rate at the exothermic peak. By assuming autocatalytic cure rate model, the summation of reaction order is taken as 2 .

$$
\begin{gathered}
H_{\mathrm{U}}=\int_{0}^{\mathrm{t}_{\mathrm{f}}}\left(\frac{\mathrm{d} Q}{\mathrm{~d} t}\right)_{\mathrm{d}} \mathrm{d} t \\
H_{\mathrm{T}}=\int_{0}^{\mathrm{t}_{\mathrm{f}}}\left(\frac{\mathrm{d} Q}{\mathrm{~d} t}\right)_{\mathrm{T}} \mathrm{d} t \\
\dot{\alpha}=\frac{\mathrm{d} \alpha}{\mathrm{d} t}=\frac{1}{H_{\mathrm{U}}}\left(\frac{\mathrm{d} Q}{\mathrm{~d} t}\right)_{\mathrm{T}} \\
\alpha=\frac{1}{H_{\mathrm{U}}} \int_{0}^{\mathrm{t}}\left(\frac{\mathrm{d} Q}{\mathrm{~d} \alpha}\right)_{\mathrm{T}} \mathrm{d} t \\
\dot{\mathrm{d} t}=\left(k_{1}+k_{2} \alpha^{\mathrm{m}}\right)(1-\alpha)^{\mathrm{n}} \\
\dot{\alpha}=\left(k^{\mathrm{m}}\right)(1-\alpha)^{\mathrm{n}} \\
\alpha_{\mathrm{P}}=\frac{m}{m+n} \\
\alpha+n=2(\text { autocatalytic }) \\
\mathrm{P}_{\mathrm{P}}=\frac{m^{\mathrm{n}} n^{\mathrm{n}}}{m+n^{(\mathrm{m}+\mathrm{n})}}
\end{gathered}
$$

\section{Experimental}

\section{Materials and methods}

Natural zeolite as an inorganic filler was obtained from the Gördes-Manisa region. The zeolite is composed of mainly clinoptilolite (64\%). The sample was ground in a laboratory type ball-mill and washed with deionized water in order to remove the surface dust and then dried at $105^{\circ} \mathrm{C}$. In this study, zeolite samples of particle size from 25 to $106 \mu \mathrm{m}$ were used. The samples were dehydrated at $150^{\circ} \mathrm{C}$ under vacuum for $24 \mathrm{~h}$ before using for epoxy the system. EPON 828 resin was mixed with phthalic anhydride hardener [65/35]. After preparation of the epoxy system, epoxy samples were mixed with $10 \%$ natural zeolite by using a magnetic stirrer for about $15 \mathrm{~min}$. The epoxy-zeolite samples were stored at $-13^{\circ} \mathrm{C}$ before use in the DSC analysis [1].

Samples consisting of 3-6 mg mixtures were placed in aluminum DSC pans and DSC curves were obtained using DSC 50, Shimadzu series. In the isothermal scans, the samples were heated to the test temperature at $20^{\circ} \mathrm{C} \mathrm{min}{ }^{-1}$ and the heat flow vs. time curve was measured at $70,90,110,130^{\circ} \mathrm{C}$. In the dynamic scanning, the samples were heated to $260^{\circ} \mathrm{C}$ at a rate of $20^{\circ} \mathrm{C} \mathrm{m^{-1 }}$. In both scanning methods, the carrier gas was nitrogen at a flow rate of $40 \mathrm{~cm}^{3} \min ^{-1}[10]$.

\section{Results and discussion}

In this work, the total heat of reaction for epoxy/natural zeolite system was determined directly from the results of dynamic scanning measurements. Figure 5 shows DSC curves of the epoxy system with and without zeolite at $20^{\circ} \mathrm{C} \mathrm{min}^{-1}$ heating rates. The total heat of reactions for epoxy and epoxy-zeolite system was determined by using Eq. (1). The value of total heat of reaction with and without zeolite was found to be 356 and $247 \mathrm{~J} \mathrm{~g}^{-1}$, respectively. Since this heat was proportional to the extent of curing, a higher degree of cure was obtained for zeolite-epoxy system.

The total amount of heat generated at different temperatures, $H_{\mathrm{T}}$, was determined from isothermal DSC measurements at $70,90,110$ and $130^{\circ} \mathrm{C}$. The values of the isothermal heat of reaction for each temperature were determined by using Eq. (2). Figures 6 and 7 show the isothermal DSC scans of the epoxy system with and without zeolite.

The isothermal cure rates for each temperature were obtained from Eq. (3). The isothermal cure rate curves for epoxy and epoxy-zeolite system at four different temperatures are shown in Figs 8 and 9. The results obtained from the isothermal cure rate curves indicate that the cure rate of the corresponding 


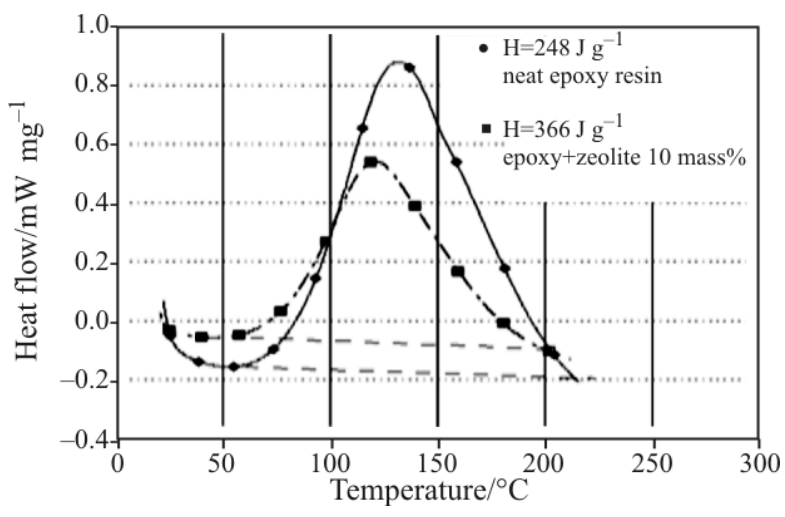

Fig. 5 DSC scans for the epoxy system with and without zeolite at heating rates of $20^{\circ} \mathrm{C} \mathrm{min}^{-1}$

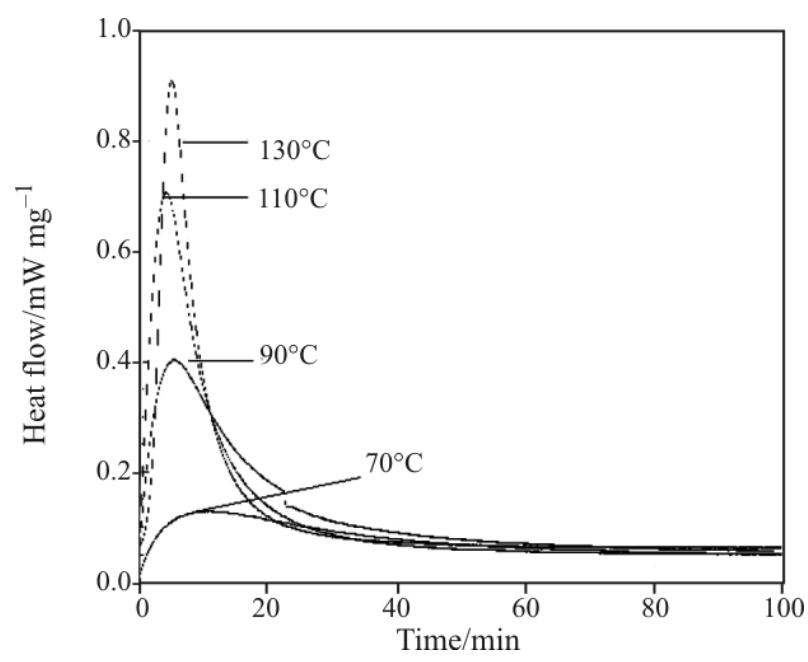

Fig. 6 Isothermal DSC scans for the epoxy system

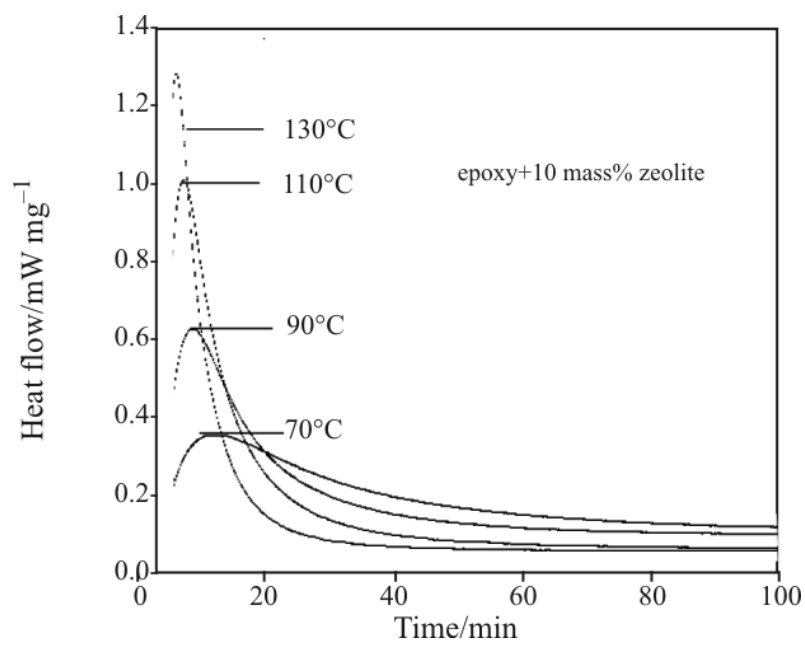

Fig. 7 Isothermal DSC scans for the epoxy system with zeolite

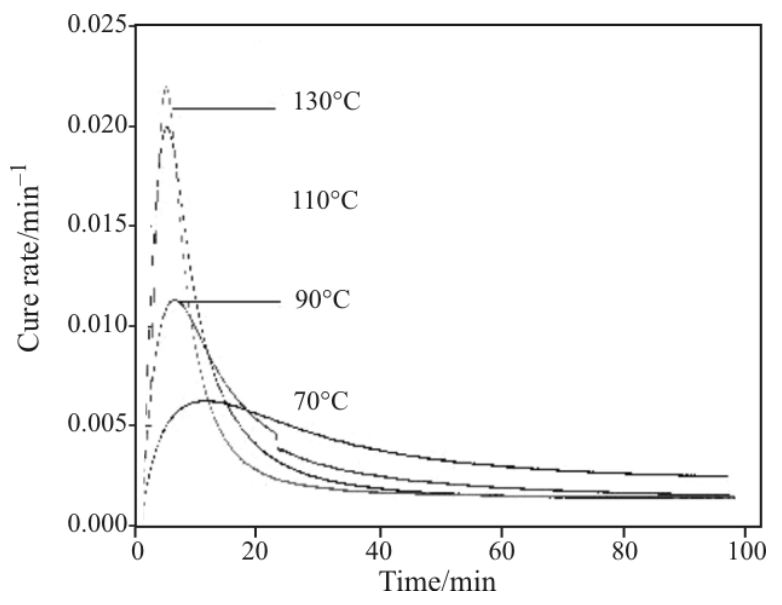

Fig. 8 Isothermal cure rate as a function of time for the epoxy system

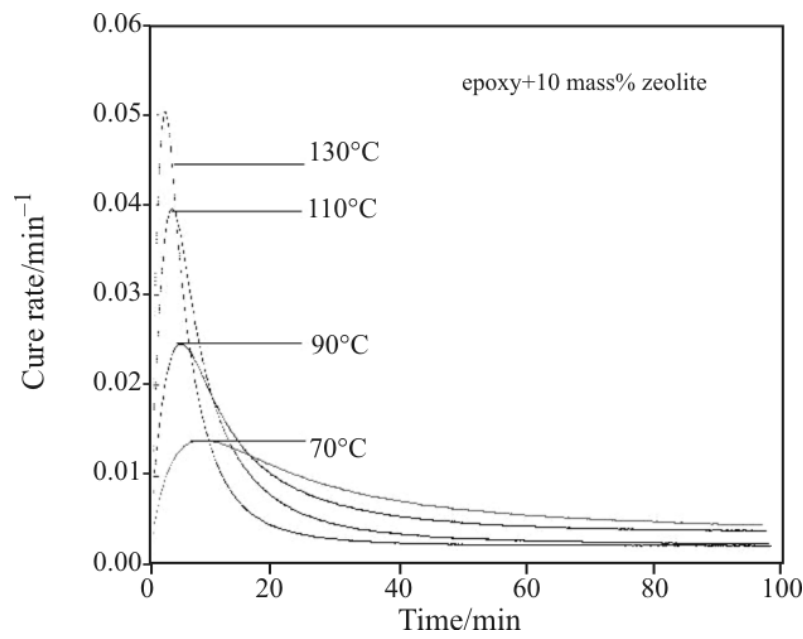

Fig. 9 Isothermal cure rate as a function of time for the epoxy-zeolite system

system increased with the increase of the temperature up to $130^{\circ} \mathrm{C}$. The temperature at maximum peak point on the curve of zeolite-epoxy system appeared in a relatively shorter time and the value of differential cure rate at the same corresponding point was higher than that of neat epoxy. In that manner, the autocatalytic reaction of the hydroxyl group was believed to play a substantial role in the occurrence of a feasible new transition state with anhydride hardener and epoxide group, all of which may promote the reaction involved.

The values of degree of cure for each temperature were obtained from Eq. (4). The results are shown in Figs 10 and 11. The predicted curves in plots of degree of cures for epoxy and epoxy-zeolite system are in sigmoid shape, proving that both systems could be reasonably interpreted by the autocatalytic cure rate expression.

The values of kinetic parameters $(m, n$ and $k$ ) with a regression constant of 0.99 were determined by simultaneously solving Eqs (7)-(9). The results of kinetic pa- 


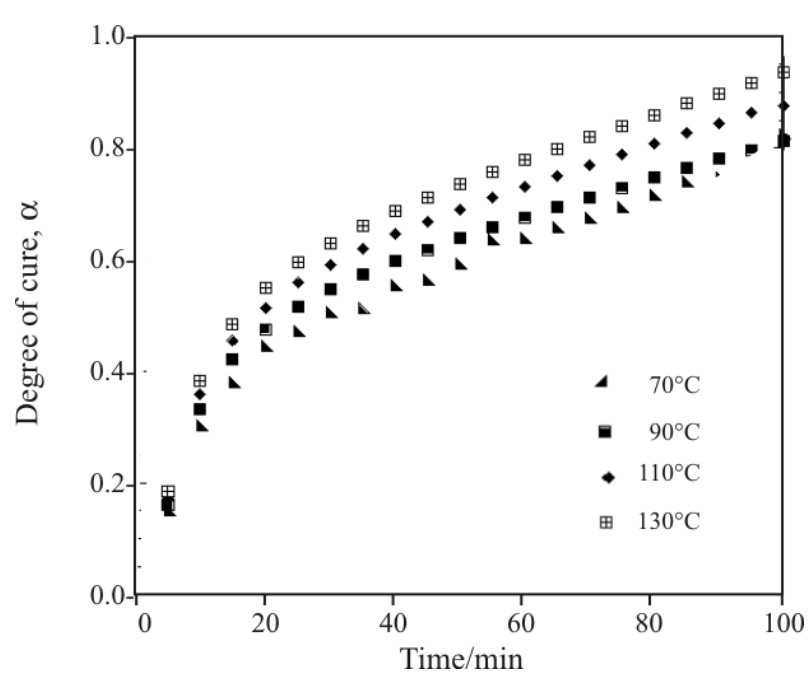

Fig. 10 Degree of cure curves at different temperatures for the epoxy system

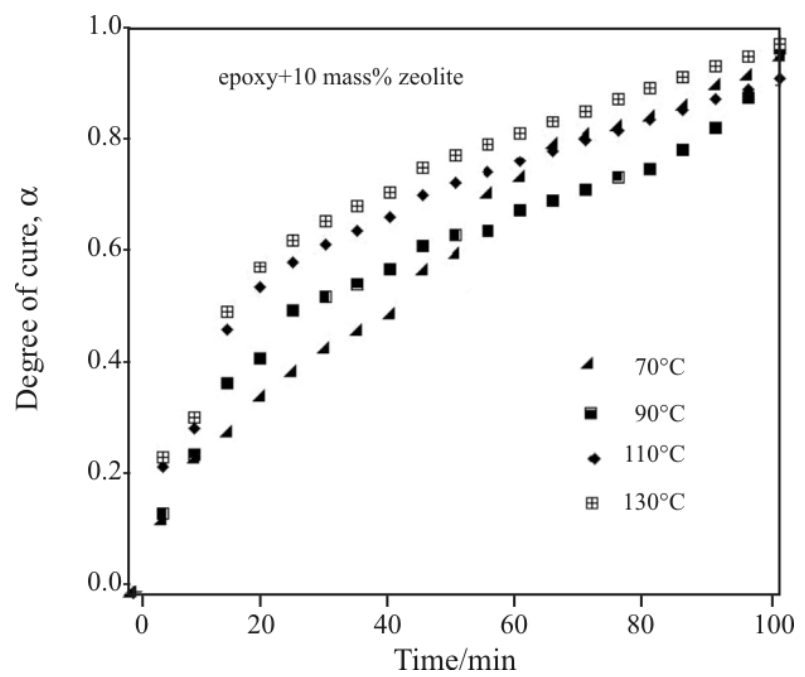

Fig. 11 Degree of cure curves at different temperatures for the epoxy-zeolite system

Table 1 Kinetic parameter for the epoxy system

\begin{tabular}{cllc}
\hline$T / \mathrm{K}$ & $m$ & $n$ & $k \cdot 10^{2} / \mathrm{min}^{-1}$ \\
\hline 343 & 1.8 & 0.2 & 1.16 \\
363 & 1.4 & 0.6 & 1.42 \\
383 & 0.82 & 1.18 & 10.2 \\
403 & 0.36 & 1.64 & 11.62 \\
\hline
\end{tabular}

Table 2 Kinetic parameter for the epoxy-zeolite system

\begin{tabular}{cccc}
\hline$T / \mathrm{K}$ & $m$ & $n$ & $k \cdot 10^{2} / \mathrm{min}^{-1}$ \\
\hline 343 & 0.44 & 1.56 & 2.2 \\
363 & 0.52 & 1.48 & 14.7 \\
383 & 0.61 & 1.39 & 20 \\
403 & 0.65 & 1.35 & 23.8 \\
\hline
\end{tabular}

rameters for epoxy and epoxy-zeolite system are tabulated in Tables 1 and 2 . The divergence in values of reaction orders ( $m$ and $n$ ) at various temperatures implied that the reaction mechanism of corresponding the epoxy resin was highly dependent on the temperature applied. It is because of the fact that epoxy kinetic mechanism includes very complex series of curing reactions, some of which only occurred at elevated temperatures. The reaction rate constant $(k)$ gave also the same response to the temperature changes. The values of rate constants at low curing temperatures demonstrated slight changes with the addition of the zeolite. Moreover, at elevated temperatures, the rate constant increased and became more pronounced for the filled system.

\section{Conclusions}

In this study, the cure kinetics of epoxy resin and epoxy resin containing 10 mass $\%$ natural zeolite were investigated by using DSC. The conformity of the cure kinetic data of epoxy and epoxy-zeolite system was checked with the autocatalytic cure rate model. The dynamic scan results revealed that the cure rate was increased by the addition of zeolite. Moreover, zeolite-epoxy system has moderately higher peak temperature when compared with the unfilled system, as a result of the zeolite particles acting as catalyst for epoxy system.

The isothermal cure rate results indicated that the cure rate increased with increasing temperature. The highest cure rates for epoxy and epoxy-zeolite system were obtained at $130^{\circ} \mathrm{C}$. Moreover, the values of cure rate for epoxy were lower than the value of cure rate for epoxy-zeolite system at each temperature. The maximum point on the isothermal cure rate appeared in shorter time for epoxy-zeolite system and the value of cure rate was higher than that of the unfilled epoxy. This behaviour was related to the autocatalytic effect. As a result, the autocatalytic reaction of the hydroxyl group on zeolite surface plays a significant role in the occurrence of the new transition state with anhydride hardener and epoxy group in the resin. Besides, the shape of the degree of cure results gave the idea about the reaction mechanism of the epoxy and epoxy-zeolite system. All curves were of sigmoid shape and both systems could be interpreted by the autocatalytic cure rate model.

The reaction orders and the reaction rate constants varied with temperature. These variations indicated that the epoxy kinetic mechanism was composed of series of reactions, some of which only occurred at high reaction temperature. The rate constant increased with increasing temperature. At higher temperature, the variation of reaction constant for epoxy-zeolite was more pronounced than that of epoxy system. 


\section{References}

1 J. Y. Lee, M. J. Shim and S. W. Kim, Mater. Chem. Phys., 48 (1997) 36.

2 D. Roşu, C. N. Çaşcaval, F. Mustata and C. Ciobanu, Thermochim. Acta, 383 (2002) 119.

3 M. S. Cabezudo, M. G. Prolongo, C. Salom and R. M. Masegosa, J. Therm. Anal. Cal., 86 (2006) 699.

4 J. Y. Lee, H. K. Choi, M. J. Shim and S. W. Kim, Thermochim. Acta, 343 (2000) 111.

5 A. Top and S. Ülkü, Appl. Clay Sci., 27 (2004) 13.

6 E. Chmielewská, L. Sabova and K. Jesenák, J. Therm. Anal. Cal., 92 (2008) 567.
7 S. Du, Z. -S. Guo, B. Zhang and Z. Wu, Polym. Int., 53 (2004) 1343.

8 M. G. Lu, M. J. Shim and S. W. Kim, J. Therm. Anal. Cal., 58 (1999) 701.

9 A. Dutta and M. E. Ryan, J. Appl. Polym. Sci., 24 (1979) 635.

10 M. Lu, M. Shim and S. Kim, Polym. Eng. Sci., 39 (1999) 274.

DOI: $10.1007 / \mathrm{s} 10973-008-9366-7$ 